\title{
Recovery Method for Emergency Situations with Hazardous Substances Emission into the Atmosphere
}

\author{
Maksym V. Kustov*, Volodymyr D. Kalugin, Olena V. Hristich, Yuliana K. Hapon
}

Special Chemistry and Chemical Technology Department, National University of Civil Defence of Ukraine, 94 Chernyshevska Str., Kharkiv 61023, Ukraine

Corresponding Author Email: m.kustov.nuczu@gmail.com

https://doi.org/10.18280/ijsse.110415

Received: 19 January 2021

Accepted: 20 July 2021

\section{Keywords:}

artificial precipitation, emergency situation, emission, hazardous substances, localization emergency zone

\begin{abstract}
The objective of this Article is development of a hazardous area extent reducing method in the atmosphere upon emergency situation occurrence with hazardous chemical and radioactive substances emission. To achieve the specified objective it is recommended to use deposition of gaseous, liquid and solid disperse hazardous particles from the atmosphere using artificial precipitation over a zone of emergency. For artificial intensification of precipitation, use of chemical and physical principles of impact on droplets formation processes in the area of clouds is proposed. A pyrotechnic composition was developed for chemical impact, which when combustion generates finely dispersed chemical centers of condensation and modifying components for hazardous chemical substances neutralization. Conducting the ionization of precipitation formation area by a strong impulse electromagnetic irradiation was proposed as a physical impact on the artificial precipitation formation processes. The laboratory study results confirmed the technical feasibility of this method implementation. To reduce the necessary radiator power, application of a multi-position radiators system with radiation focusing to one irradiation zone was proposed. The theoretical and experimental results obtained in the article are the base for development of emergency situation negative consequences reduction practical procedures through artificial precipitation initiation.
\end{abstract}

\section{INTRODUCTION}

Artificial initiation of precipitation over a certain territory allows finding a solution to a number of crucial tasks. They include ensuring fire safety - extinguishing of large-scale natural fires and fire hazard reducing of the forest, steppe and peat areas; environmental safety - purification of atmosphere against hazardous toxic and radioactive substances and atmospheric pollution areas reducing upon various natural and man-caused accidents; social safety - provisioning population and agricultural lands with water.

Resulting from large natural and man-caused fires a large amount of combustion products is emitted into the atmosphere. Due to the air masses motion, the combustion products are spread over dozens of kilometers [1]. When contact with the atmosphere constituents, some gases, generated when combustion, react with them with new compounds generation capable of condensation under the atmospheric conditions [2]. To forecast the behavior of hazardous combustion products in the atmosphere along with gaseous and solid aerosol components one has to consider the peculiarities of liquid aerosols generation and excretion from the atmosphere [3].

Under normal operation of industrial enterprises, a large amount of hazardous chemical substances are emitted into the environment. Particularly large-scale emissions occur upon emergencies arising at such facilities [4]. The greatest hazard for the ecosystem is represented by atmospheric emissions of chemically hazard pollutants $[5,6]$.
Upon the occurrence of large-scale emergencies at the atomic energetics facilities, a radioactive substances emission into the atmosphere is possible. The most large-scale emergencies in history are disasters at the Chernobyl Nuclear Plant in Ukraine [7] and the Fukushima Nuclear Plant in Japan [8]. Resulting from emergency depressurization of nuclear reactor a large amount of gaseous and aerosol radioactive substances are released into the atmosphere. Being affected by convection aerial currents radioactive substances penetrate the upper layers of the troposphere and spread over large distances [9].

To date, localization and elimination of the consequences of technogenic accidents with the release of hazardous substances into the atmosphere is carried out by organizing water spraying [10]. However, due to the large size of the contaminated zone, this presents significant difficulties and requires the involvement of a large amount of manpower and resources.

Precipitation over an emergency area allows significantly reduce the pollution area extent and the overall radiation background [11]. This occurs due to radionuclides sorption by water droplets. However, solving the problem of active influence on precipitation requires some significant amount of energy and taking into account of various meteorological factors affecting the precipitation formation process. The main factors determining the precipitation formation process include air humidity and condensation centers availability. 
Liquid microdroplets of water and aqueous solutions of salts and acids, solid particles of dust and soot as well as ions can serve as the nuclei.

Currently, finely dispersed solid $\mathrm{CO}_{2}$, salts $\mathrm{NaCl}$ and $\mathrm{AgCl}$ are actively used. By modeling the effect of the physicochemical properties of reagents on the dynamics of the sedimentation process, effective reagents and new compositions are being actively developed [12]. Besides the physical and chemical properties of reagents introduced, their dispersiveness plays an important role. A significant impact on the precipitation formation process is caused by the introduction method of reagent aerosol particles to the zone of activation - this is the use of specially equipped aircraft [13] and small missiles [14].

The greatest activity to vapour condensation is possessed by charged ions and ion clusters [15]. Based on this, artificial increase in ions concentration in the atmospheric area over a fire zone will allow intensifying precipitation in this region. Under normal conditions, about $10^{3} \mathrm{~cm}^{-3}$ of light ions are contained in the atmosphere. Chemically active salts $(\mathrm{AgCl})$ are introduced to the atmosphere to increase the number of condensation nuclei [16].

It has been established that well-developed convective clouds with a thickness (thickness) of at least $2.5 \mathrm{~km}$ with a temperature at the upper limit of not more than $-6^{\circ} \mathrm{C}$ are suitable for artificial precipitation [17]. Rainfall begins to fall from the cloud 8-12 min after the introduction of the reagent and, depending on the power and water supply of the cloud, lasts 20-50 min or more. Guo et al. [18] propose the use of the thermoionization effect, which consists in the flow of thermoelectron emission of free electrons from a surface heated to high temperatures $(\mathrm{T} \approx 1900-2000 \mathrm{~K})$, products of squib combustion used for active effects on clouds.

Based on this process, was developed a technique of woods fires extinguishing at the expense of artificial initiating of precipitation by reagents spraying by aircraft [19]. However, this method only works under some particular meteorological conditions (high humidity, low temperature, etc.)

Taking into account the fact that charged particles are more active than salts, introducing ions to the influence zone instead of salts will allow broadening the application conditions of the artificial precipitation intensification.

In ref. [20, 21], high intensity of droplet formation was theoretically and experimentally demonstrated when free ions were introduced into the zone of influence. However, the issue of delivery of such ions at an altitude of $3.5 \mathrm{~km}$, in the zone of active precipitation, remains unresolved. When emitting chemically hazardous substances, in addition to precipitation, it is necessary to investigate the issue of their chemical neutralization.

Therefore, the aim of this work is to solve the problem of artificial control of sedimentation processes in a wide range of meteorological conditions using chemical and electrophysical methods, as well as modification of pyrotechnic compositions for effective precipitation and neutralization of hazardous substances.

\section{MATERIALS AND METHODS OF RESEARCH}

\subsection{Materials of research}

On the results of classification and systemizing the hazardous substances penetrating the atmosphere when a natural or man-caused emergency situation, the main objects of deposition were determined to minimize the negative consequences for the atmosphere of various nature emergencies. The results are systemized in Table 1.

Table 1. The main negative factors for the atmosphere during emergencies of various kinds

\begin{tabular}{ccc}
\hline $\begin{array}{c}\text { Man-made } \\
\text { accidents without } \\
\text { fires }\end{array}$ & Large-scale fires & $\begin{array}{c}\text { Accidents at } \\
\text { nuclear facilities }\end{array}$ \\
\hline Ammonia $\left(\mathrm{NH}_{3}\right)$ & Soot $\left(\mathrm{C}, \mathrm{CO}_{\mathrm{x}}\right)$ & $\begin{array}{c}\text { Radioactive water } \\
\text { aerosol }\end{array}$ \\
Chlorine $\left(\mathrm{Cl}_{2}\right)$ & $\begin{array}{c}\text { Ash (oxides of } \\
\text { alkali and alkaline } \\
\text { earth metals) }\end{array}$ & $\begin{array}{c}\text { Aerosol of } \\
\text { radioactive solid } \\
\text { inorganic particles } \\
\text { Radioactive }\end{array}$ \\
$\begin{array}{c}\text { Hydrocyanic acid } \\
(\mathrm{HCN})\end{array}$ & $\begin{array}{c}\text { Sulfur oxides } \\
\left(\mathrm{SO}_{\mathrm{x}}\right)^{*}\end{array}$ & $\begin{array}{c}\text { chemically inert "hot } \\
\text { particles" }\end{array}$ \\
\hline
\end{tabular}

\subsection{Methods of research}

Upon considering the hazardous particles deposition processes from the atmosphere, the fact that hazardous gaseous particles removal occurs according to the pattern of phase-by-phase gas absorption by rain droplet, and disperse particles deposition occurs under the mechanism of gravity coagulation is born in mind.

According to the model proposed [22], the process of pollutant atmospheric gases washing-out by water droplets can be divided into several individual stages. Schematic representation of these processes is in Figure 1.

Contents of the stages:

1. Gas molecules $\left(\mathrm{G}_{\mathrm{A}}\right)$ transporting to the droplet surface by diffusion in the gas phase;

2. Gas molecules $\left(\mathrm{G}_{\mathrm{A}}\right)$ absorption by the droplet surface and achieving the balance in the local area of discontinuity surface due to gas desorption;

3. Absorbed gas molecules $\left(\mathrm{G}_{\mathrm{A}}\right)$ transporting to the droplet volume due to diffusion in liquid;

4. Chemical reactions of absorbed gas $\left(\mathrm{G}_{\mathrm{A}}\right)$ and water in the droplet volume with reaction products formation $\left(\mathrm{G}_{\mathrm{B}}\right)$;

5. Reaction products $\left(\mathrm{G}_{\mathrm{B}}\right)$ transporting to the droplet volume;

6. Balance achieving in the droplet surface local volume due to chemical reaction products absorption-desorption;

7. Products molecules $\left(\mathrm{G}_{\mathrm{B}}\right)$ transporting from the droplet surface to the gas phase due to diffusion.

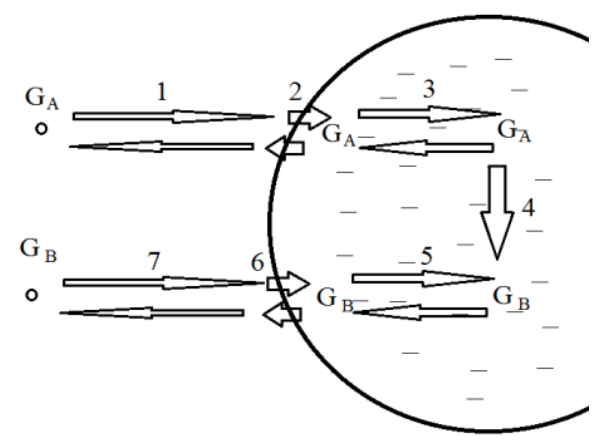

Figure 1. Diagram of chemically hazard gases stages of absorption by atmospheric aerosols droplets

The gravity coagulation mechanism essence lies in finely dispersed particles capturing by a large rain droplet when 
falling. Fine aerosol particles with radius of $r_{p}$ and concentration $\mathrm{C}_{\mathrm{p}}$ hover in the air under the influence of aerial currents $\left(\mathrm{V}_{0} \approx 0\right)$. Under the influence of the gravity force, precipitation droplets with a size of $r_{d}$ move downwards with a certain velocity $\mathrm{V}_{\mathrm{d}}$. When falling, a large droplet is air-flown, which captures fine aerosol particles. However, since the aerosol particles mass is different from zero, they are affected by the inertial forces, which tend to maintain the rectilinear trajectory. The collision probability of a large droplet with a small one (capture coefficient $\mathrm{K}_{\mathrm{g}}$ ) depends on their mutual sizes, medium viscosity $(\eta)$ and motion velocity (Figure 2 ).

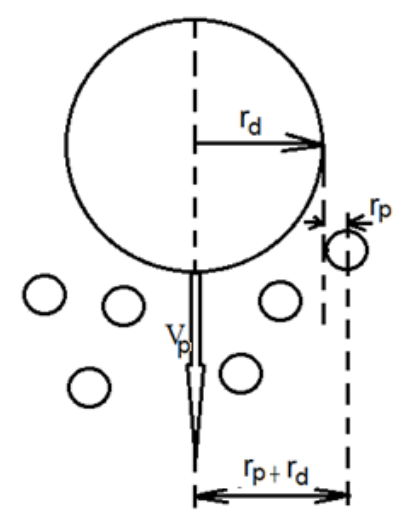

Figure 2. Disperse particles capturing diagram by precipitation droplets when gravity coagulation

Description of this process was considered in works [23, 24].

A climate chamber was developed and created to conduct experimental research on the artificial impact on the atmospheric processes.

The need for creating the reduced pressure conditions $(\mathrm{P}=54$ $\mathrm{kPa})$ in the unit determines the rigid-frame sealed arrangement of the chamber. To ensure the possibility of studying the electromagnetic irradiation effect on atmospheric aerosols, the chamber walls have been carried out in the metal design. The chamber volume is $0.5 \mathrm{~m}^{3}$. The working chamber small volume allows placing it in technical refrigeration chambers to create and maintain low temperatures in the chamber (down to $-20^{\circ} \mathrm{C}$ ) during a long-term conducting series of experiment.

The elements layout in the unit is shown in the diagram (Figure 3). An ultrasonic liquid disperser 1, which provides for dispersity of $\sim 10 \mu \mathrm{m}$ is used as aerosol. Pressure, temperature and humidity control is exercised using laboratory meteorological station 2. The droplets formation and droplets growth process is registered by type 3 LGN 2076 helium-neon laser with wavelength $-0,83 \mu \mathrm{m}$, irradiation of which is introduced to the chamber through window 4 and directed through the active impact zone to photocathode of the control photodetector 5 , in capacity of which acts the PMT-79 type photomultiplier tube with photocathode sensitivity - 120 $\mu \mathrm{A} / \mathrm{lm}$. In the upper part, a closable hole 6 is provided for dispersed chemical reagents introducing to implement the artificial precipitation formation process by the chemical impact method. When studying atmospheric aqua-aerosols deposition by the electromagnetic method, microwave range radiation generator 7 is installed on the chamber bottom in the form of pulsed magnetron with the capability to change the impulse duration from $0.05 \mu$ s to $1 \mu$ s. The microwave generator operating frequency is $10 \mathrm{GHz}$, which allows creating the necessary electromagnetic field strength in the zone of microwave -radiation direct effect for aerial environment ionization with subsequent droplet formation process. The impulse duration and radiator output power adjustment is carried out using controller 8 .

Based on the fact that the main mechanism of precipitation formation intensification by electromagnetic irradiation is vapour condensation and droplets coagulation on ions, the unit is equipped with Sapfir-3K10 aeroion counter, the sensitivity of which is up to $200 \mathrm{un} \cdot \mathrm{m}^{-3}$. To determine the electromagnetic field strength in the zone of microwave radiation effect, the generator is beforehand calibrated as per the impulse length and output power using an electromagnetic field strength meter TES-593, frequency range $10 \mathrm{MGz}-8 \mathrm{GGz}$.

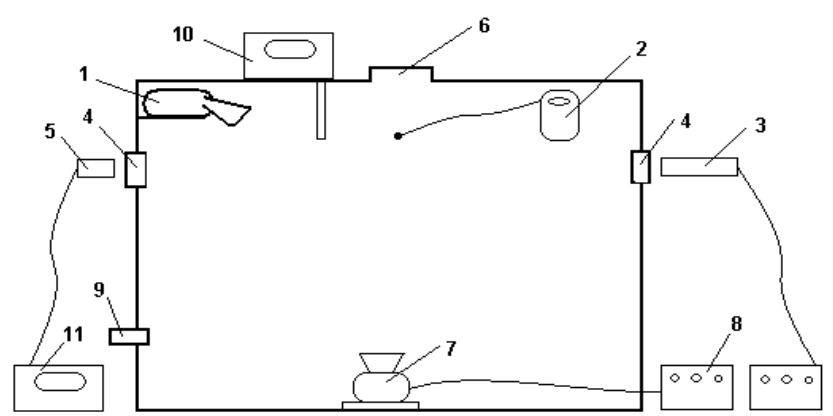

1 - ultrasonic liquid disperser; 2 - laboratory meteorological station; 3 - laser; 4 -window; 5 -photodetector; 6 - chemical reagent input hole; 7 - microwave range irradiation generator; 8 - controller; 9 - air exhaust connector; 10 aeroions counter; 11 - photomultiplier tube control unit

Figure 3. Diagram of laboratory unit for processes modelling in atmospheric aerosols

The unit represented in Figure 3 allows changing the aerial environment physical parameters in a wide range depending on the simulated atmospheric layer height. The proposed control methods ensure the possibility of effectively researching the size of droplets and their growth dynamics. Using this unit makes it possible to study the influence processes on the atmospheric seeding aerosols of deposition zone with chemically active salts, cooling agent spraying, electromagnetic and ultrasonic irradiation, corona discharge, etc.

The experiment methodology is as follows: the chamber is filled with liquid aerosol formed by the ultrasonic disperser 1 . The ultrasonic frequency variation on the disperser diaphragm allows varying the liquid aerosol dispersity in the range of 5$30 \mu \mathrm{m}$. Water with the same volume was dispersed into the chamber. In the chamber upper angle, an explosive charge of the above dimensions was placed on-mount. The watery aerosol deposition time when the explosive charge combustion was compared with the background time of deposition without exercising any effect.

\section{PECULIARITIES OF ELIMINATION EMERGENCIES WITH HAZARDOUS SUBSTANCES EMISSION BY ARTIFICIAL INITIATING PRECIPITATION}

Upon the occurrence of natural or man-caused nature emergencies, hazardous emissions into the atmosphere are spread by convective currents at heights up to $1 \mathrm{~km}$, wherein the existing emergencies consequences minimizing methods and ways for the atmospheric air are capable to influence the contamination zone at heights up to $20 \mathrm{~m}$. Accordingly, there 
is a significant contradiction between the atmospheric air contamination zone and the zone of emergency consequences active minimizing for the atmospheric air. To solve the tasks specified one has to develop a physical model of emergencies consequences elimination process for the atmosphere by the methods of artificial precipitation formation. The developed method is based on the chemical and radioactive pollutants in gaseous, liquid and solid aerosol states sorption principles by atmospheric precipitation falling droplets. Herewith, the lower part of the atmosphere up to $5 \mathrm{~km}$ high is broken down into two conventional zones (Figure 4).

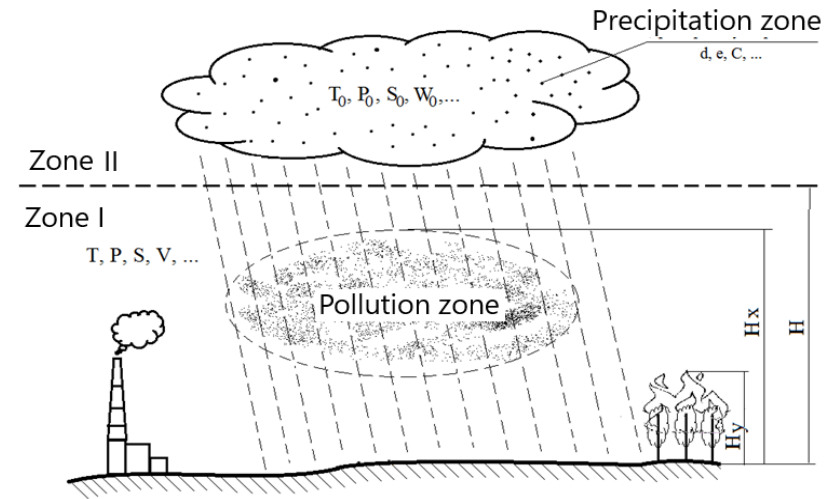

Figure 4. General diagram of emergency consequences elimination methods for the atmospheric air by artificial precipitation initiating

The zone of the atmospheric air contamination by emergency is attributed to the first zone. The damage area boundaries are determined by the maximum allowed concentration exceedance boundaries for chemically hazardous substances and the exposure dose sanitary regulations for radioactive contamination.

At a man-caused emergency, a chemical or radiation damage cloud is formed in the atmosphere, which increases intensively till the emission source has been contained. A significant part of gaseous and aerosol products of combustion is emitted into the atmosphere when large-scale natural fires occurrence. The emission intensity depends on the intensity and area of burning. An essential factor when pollutants deposition is their physical and chemical properties analyzed below.

Due to horizontal and vertical atmospheric currents, the damage area is spread quickly, which stipulates the need for prompt forecasting the damage area depending on the wind velocity and direction. According to its area, the zone I coincides with the actual one and the forecast damage areas and is spread heightwise from the Earth surface to the lower cloudiness boundary. The zone I can contain chemical and radiation contamination clouds as well as natural fire territory as a powerful source of hazardous substances emission into the atmosphere. Various intensity precipitation droplets, when flying through the zone I, are partially or fully evaporated; absorb hazardous substances depositing them on the soil surface; increase the air humidity and combustible substances reducing the fire intensity.

Another zone is the cloudiness zone from the lower to the upper boundary of clouds potentially capable of precipitation formation. Depending on the meteorological conditions the heights of the upper and lower boundaries of II zone can vary. This is this the zone where the precipitation formation process occurs, which is affected by meteorological conditions (pressure $\mathrm{P}_{0}$, temperature $\mathrm{T}_{0}$, humidity $\mathrm{S}_{0}$ and moisture content in cloud $\mathrm{W}_{0}$, etc.) and natural or artificial droplet formation nuclei properties (dispersity d, concentration $\mathrm{C}$, electric charge e, etc.). However, not all kinds of clouds have the precipitation formation potential upon artificial impact on them. Assessment of the precipitation formation potential needs to be considered in more detail.

When considering the artificial precipitation initiating issues, important characteristics of clouds are their water and moisture content. Yet water content in the condensed state is always much less than in vaporous one.

Determining the water and moisture content is carried out using land-based and aerial monitoring means. The land-based include automatic and controlled meteorological radar systems, which operate under the principle of examined zone electromagnetic irradiation and reflected signal reception.

Rain droplets are of 0.5 to $5 \mathrm{~m}$ in diameter. Large size droplets when falling divide into smaller ones due to wind resistance. Drizzling droplets are characterized by sizes in the range of $0.05-0.5 \mathrm{~mm}$. Natural precipitation is featured by droplet falling velocity, according to their size.

Potentially suitable for artificial impact are the low-level clouds, among which the most water and moisture content have nimbostratus $(\mathrm{Ns})$ and cumulonimbus $(\mathrm{Cb})$ clouds. Other kinds of clouds are capable of precipitation formation only after the phase of their transformation into $\mathrm{Ns}$ or $\mathrm{Cb}$ kinds.

\section{STUDYING THE ARTIFICIAL INFLUENCING THE PRECIPITATION FORMATION PROCESSES}

\subsection{Chemical impact on the precipitation formation process}

All pyrotechnic compounds for artificial precipitation initiating consist of four component classes depending on their functional purpose (Figure 5).

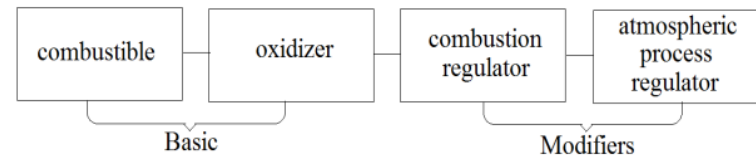

Figure 5. Main classes of pyrotechnic compounds components

Selection of the most effective substance as an explosive charge component shall be considered in conjunction with other components because a general chemical reaction of all charge components combustion takes place at the working stage.

The first factor determining the chemical composition of reagent for artificial precipitation formation is the initiated precipitation aggregative state, i.e. orientation for the process of solid-phase ice formation or liquid-phase droplet formation taking into account the Thomson equation:

$$
\frac{2 \sigma M}{\rho N_{A}} \cdot \frac{1}{r}=k T \ln \frac{p}{p_{H}}
$$

where, $r$ - generated nucleation center radius; $\sigma$ - nucleation center surface energy; $\mathrm{k}$ - Boltzmann constant; $\mathrm{T}$ - nucleation center temperature; $\mathrm{M}$ - water molecular mass; $\rho$ - droplet density; $\mathrm{N}_{\mathrm{A}}-$ Avogadro's number; $\mathrm{p}$ - air pressure at a large 
distance from droplet (crystal); $\mathrm{p}_{\mathrm{H}}-$ saturated steam pressure over droplet (crystal).

$\mathrm{AgJ}$ is used as a crystal-forming reagent in the practice of precipitation formation. There is no uniform theoretical approach of explaining its high crystal-forming effectiveness, however, the main theoretical prerequisite of the water crystallization process on the surface of $\mathrm{AgJ}$ is structures similitude of AgJ salt and ice crystals crystal gratings. The reagent crystal-forming activity, besides the chemical nature, is determined by the particle dispersity as well.

Let us consider burning temperature and rate (burning temperature and rate determine draught and, accordingly, reagents spraying) as the main parameters of explosive charge combustion. Below analyzed the main factors affecting the combustion process as well as existing models for this process description.

Ammonium perchlorate $\left(\mathrm{NH}_{4} \mathrm{ClO}_{4}\right)$ and ammonium nitrate $\left(\mathrm{NH}_{4} \mathrm{NO}_{3}\right)$ are mainly used as oxidizer in solid-state pyrotechnic compounds. Ammonium perchlorate has the greatest oxidative activity, yet harmful halogenide $\mathrm{HCl}$ is generated in the process of its combustion. Replacement of $\mathrm{NH}_{4} \mathrm{ClO}_{4}$ by $\mathrm{NH}_{4} \mathrm{NO}_{3}$ leads to an abrupt decrease in burning rate and reactive thrust.

Alkaline and alkaline-earth metals are used as combustibles in pyrotechnic charges. The most accessible ones include magnesium $(\mathrm{Mg})$ and aluminum $(\mathrm{Al})$.

Let us examine the main combustible properties of pyrotechnic compounds of $\mathrm{Al}$ and $\mathrm{Mg}$ as combustible, and $\mathrm{NH}_{4} \mathrm{ClO}_{4}, \mathrm{NH}_{4} \mathrm{NO}_{3}$ and $\mathrm{KNO}_{3}$ as oxidizer. As it was mentioned above, for safety reasons, it is reasonable to use ammonium or potassium nitrates as an oxidizer.

As of today, there are two approaches available to develop new pyrotechnic compounds for artificial precipitation initiation. The first approach is based on the fact that the main substances - water condensation and coagulation processes activators - are present in pyrotechnic compound as additives, and do not take part in combustion chemical reaction. In another approach, the main active nuclei of condensation are formed in the result of the chemical reaction of primary components combustion.

One of the control methods against acid precipitation was proposed the acid neutralisation method before its penetration the Earth's surface through finely dispersed alkali spraying. Fine dispersed alkali introduction into the atmosphere can be carried out by using the above pyrotechnic compounds.

The most accessible alkalies are sodium and potassium hydroxides $(\mathrm{NaOH}, \mathrm{KOH})$. In addition, these alkalies are well dissolved in water by decomposing into ions, which leads to their high activity when water-acid solutions neutralizing. To unify the compounds, the components of the above specified explosive charges (PC-3-71) are considered as the main combustible components of pyrotechnic compound for alkali spraying. Compound PC-4-0: Magnesium powder - $4-7 \%$; Aluminum powder $-4-4 \% ; \mathrm{KNO}_{3}-79 \%$; Bonding resin - 26 $-10 \%$.

Fine disperse alkalies $(\mathrm{NaOH}, \mathrm{KOH})$, as explosive charge fillers, do not take part in the process of combustion. In the result of explosive charge combustion the alkali particles are sprayed, contacting with acidic atmospheric formations and neutralize them by reactions (2-5):

$$
\begin{gathered}
\mathrm{H}_{2} \mathrm{SO}_{4}+2 \mathrm{NaOH} \rightarrow \mathrm{Na}_{2} \mathrm{SO}_{4}+2 \mathrm{H}_{2} \mathrm{O} \\
\mathrm{H}_{2} \mathrm{SO}_{4}+2 \mathrm{KOH} \rightarrow \mathrm{K}_{2} \mathrm{SO}_{4}+2 \mathrm{H}_{2} \mathrm{O}
\end{gathered}
$$

$$
\begin{gathered}
\mathrm{HNO}_{3}+\mathrm{NaOH} \rightarrow \mathrm{NaNO}_{3}+\mathrm{H}_{2} \mathrm{O} \\
\mathrm{HNO}_{3}+\mathrm{KOH} \rightarrow \mathrm{KNO}_{3}+\mathrm{H}_{2} \mathrm{O}
\end{gathered}
$$

Since the specified alkali have a relatively low boiling temperature $\left(\mathrm{T}_{\text {boil }}(\mathrm{NaOH})-1676 \mathrm{~K} ; \mathrm{T}_{\text {boil }}(\mathrm{KOH})-1600 \mathrm{~K}\right.$.), in the process of explosive charge combustion their sublimation occurs, which leads to additional dispersion of alkali particles.

To select the explosive charge components one has to determine the mass composition.

Determining the mass composition of effective was conducted experimentally, by determining the explosive charge combustion temperature and burnout time.

The composition is formed in the form of cylindric extended candle with diameter of $\mathrm{d}=20 \mathrm{~mm}$ and length of $1=150 \mathrm{~mm}$.

The explosive charge ignition occurs by the electrospark method. Combustion rate was determined by burn-through time measuring between control marks. The combustion temperature was determined using a high-temperature pyrometer «Flus», IR866. The experiment was conducted in an isolated chamber (Figure 6).

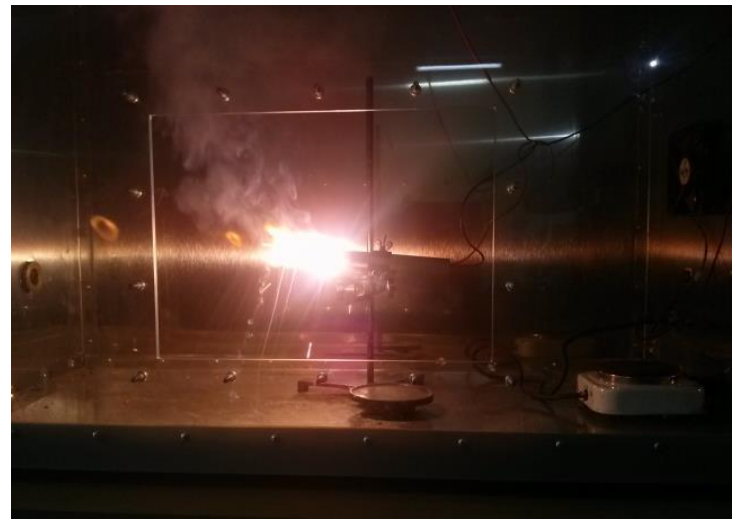

Figure 6. Experimental study of explosive charges (squib) combustion parameters

In the result of experiments, a low probability of explosive charges ignition by the electrospark method was established. This negative phenomenon is also related to the oxide film presence, which prevents the combustion process initiation. To rectify this disadvantage use a two-layer charge structure with a thin layer of initiating composition based on potassium and graphite $\left(\mathrm{KClO}_{4}+\mathrm{C}\right)$ perchlorate in the zone of ignition and main working composition using potassium nitrate and metals was proposed (Figure 7).

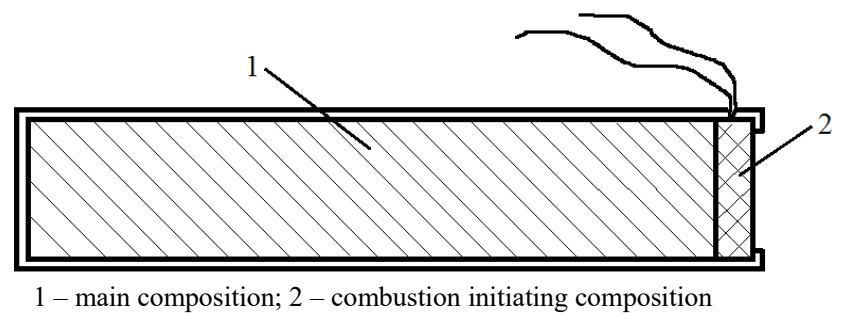

Figure 7. Two-layer explosive charge (squib) structure

The two-layer composition test results show that the changes introduced do not affect the combustion intensity within the measurement error. Herewith, the probability of ignition in all series amounted to $100 \%$.

Experimental research results are given in Table 2. 
Table 2. Main combustion parameters of pyrotechnic compounds for acidity neutralization with cellulose adding

\begin{tabular}{|c|c|c|c|c|}
\hline Com-pound & Content KOH, \% (mass) & Cellulose content, \% (mass) & Burnout rate, $10^{3} \mathrm{~m} \cdot \mathrm{s}^{-1}$ & Combustion temperature, $\mathrm{K}$ \\
\hline PC-4-51 & 50 & 5 & $2,4 \pm 0,1$ & $1790 \pm 20$ \\
\hline PC $-4-52$ & 50 & 10 & $2,4 \pm 0,1$ & $1780 \pm 20$ \\
\hline PC $-4-53$ & 50 & 15 & $2,4 \pm 0,1$ & $1760 \pm 20$ \\
\hline PC -4-61 & 60 & 5 & $2,1 \pm 0,1$ & $1750 \pm 20$ \\
\hline PC -4-62 & 60 & 10 & (flame blowoff) & $1720 \pm 20$ \\
\hline PC -4-63 & 60 & 15 & (flame blowoff) & $1710 \pm 20$ \\
\hline PC -4-71 & 70 & 5 & (flame blowoff) & $1700 \pm 20$ \\
\hline
\end{tabular}

\subsection{Physical impact on the precipitation formation process}

The most active droplet formation centers in the atmosphere are charged particles (ions). Besides artificial introducing of condensation charged nuclei using chemical reagents, there are various electrophysical methods of the air components artificial ionization.

Causing effect on an intense electromagnetic irradiation local volume is a prospective method of the artificial ionization of air [25]. The essence of this method is that the air ionization occurs due to electromagnetic irradiation energy transfer from land-based sources to free molecules and electrons in the zone of influence. An advantage of this method is the possibility to create additional ions at a significant distance from radiator. The ionized area is foreseen to be created due to high-frequency air breakdown. Focused radio waves bundles are used for breakdown and subsequent maintaining of ionization. Ionization occurs in the bundles crossing area. The breakdown is carried out by a short radio impulse.

Air ionizing at the height of cloud formation (1-5 km) over an area up to $10 \mathrm{~km}^{2}$ is principally possible by the influence zone irradiation with strong electromagnetic irradiation bundles (Figure 8).

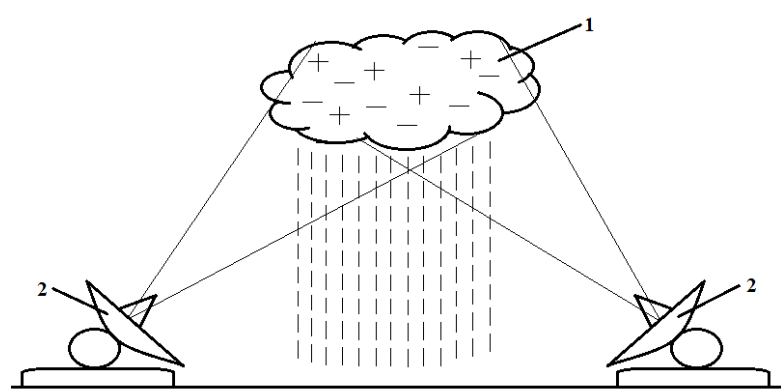

1 - active influence zone; 2 - radiators

Figure 8. Diagram of remote influence on the precipitation formation processes at the expense of electromagnetic irradiation

In the electromagnetic irradiation field, the free electrons total concentration increases virtually by two orders. Also, due to the fact that in the electromagnetic field the electrons energy drop rate is much lower than in unexcited troposphere, the electromagnetic irradiation causes affects the increase in specifically the high-energy electrons concentration [26]. Herewith, the concentration of high-energy electrons increased by $3-4$ orders at field strength $\mathrm{E}=(1.2-1.5) \mathrm{kV} \cdot \mathrm{m}^{-1}$.

To provide for the necessary field strength at heights of 1-5 $\mathrm{km}$, some strong enough sources of microwave radiation are required. In case of using reduced strength sources, let us consider the case of a multi-position radiators system (MPRS) application for artificial ionization of a specified area in the troposphere.
In case of determining the geometrical characteristics of the irradiation zone, taking into consideration of the radiators directive diagrams is mandatory (Figure 9).

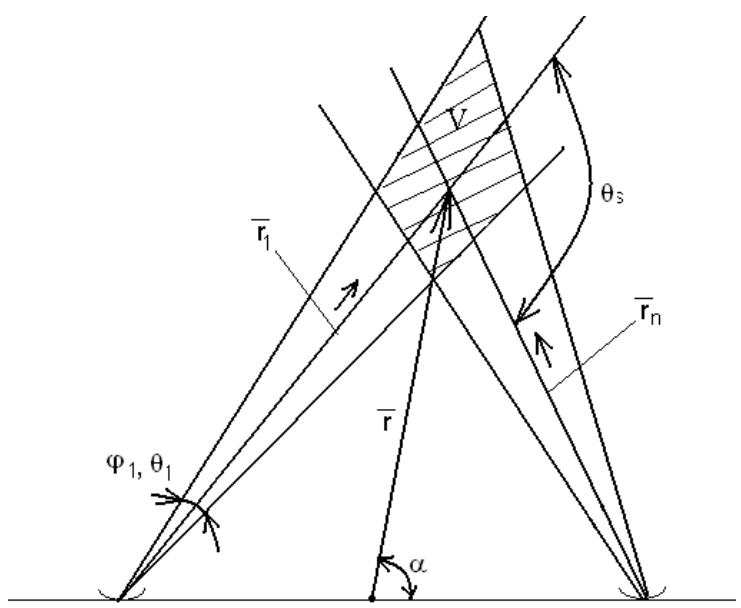

Figure 9. Geometrical characteristics of the MPRS irradiation zone

Let us consider that the directive diagram as per the radiator strength is described by the Gaussian function:

$$
\mathrm{G}_{\mathrm{n}}=\mathrm{G}_{0} \exp \left[-(\ln 2)\left[\left(\frac{2 \phi_{n}}{\alpha}\right)^{2}+\left(\frac{2 \phi_{n}}{\theta}\right)^{2}\right]\right]
$$

where, $\mathrm{G}_{0}$ - radiator action directivity factor by vector $\bar{r}_{\mathrm{n}} ; \varphi_{\mathrm{n}}$, $\theta_{\mathrm{n}}-$ angles from $\bar{r}_{\mathrm{n}}$, which determine the ray width in vertical and horizontal directions (for axisymmetric ray $\varphi_{n}=\theta_{n}$ ); $\theta_{\mathrm{s}}-$ rays crossing angle; $\alpha$ - rays crossing area center inclination angle from the center MPRS.

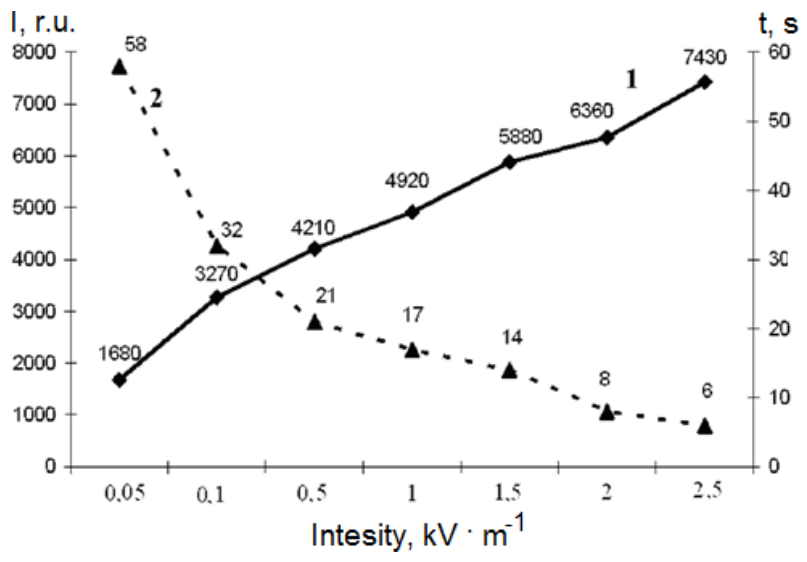

Figure 10. Diffused light intensity dependence I (curve 1) and vapour deposition time $\tau$ (curve 2 ) on the field strength in the chamber 
The research of dynamics dependence of the nucleation on ions process from microwave radiation electromagnetic field strength is of particular interest. Two parameters were controlled in the result of the experiment: diffused light intensity in peak and complete deposition time of vapour. Data on the field strength influence on the water vapour nucleation process on aeroions obtained by averaging the cycle of 3-4 measurements for each value of microwave field strength (Figure 10). The aeroions concentration variation registration was not conducted by the instrument method because of the technical complexity of probe sampling from a flask with discharge. However, based on the Wilson chamber operation principle, the relative concentration of ions can be controlled by the intensity of light diffused.

\subsection{Discussion}

The results of an experiment with pyrotechnic compounds showed that adding potassium alkali up to $60-65 \%$ (mass) allows maintaining sustainable combustion of pyrotechnic compound. Adding cellulose decreases the combustible properties of explosive charge in the result of a certain amount of active combustible components replacement with cellulose. However, the PC-4-61 compound with $60 \% \mathrm{KOH}$ and $5 \%$ of cellulose is characterized by stable combustion and can be used when acidic precipitation neutralization. In addition, magnesium and aluminum oxides, generated in the process of combustion, are the droplet formation centers, which leads to additional initiating of the precipitation formation process. Additional accelerating the process of precipitation formation can be done by introducing $\mathrm{AgI}$ or $\mathrm{NaCl}$ to pyrotechnic compound, assuming that the modifying additives total amount shall not exceed $65 \%$ of the mass ones.

Use of the proposed compound for artificial precipitation formation allows initiating precipitation over an area of largescale natural and man-caused fires, which leads to harmful combustion products deposition from the atmosphere and prevents from acidic atmospheric precipitation formation by their neutralization.

Sodium hydroxide is an effective neutralizer of chlorine:

$$
\mathrm{Cl}_{2}+2 \mathrm{NaOH} \rightarrow \mathrm{NaCl}+\mathrm{NaOCl}+\mathrm{H}_{2} \mathrm{O}
$$

To neutralize $1 \mathrm{t}$. of chlorine, $1.2 \mathrm{t}$. of $\mathrm{NaOH}$ is needed. Sodium hydroxide is well soluble in water, so water solutions are usually used in the degassing practice. As can be seen from (8), when chlorine neutralizing with alkali, salts that desublimate to hygroscopic crystals and water are additionally generated. Generation of these products of reaction (hygroscopic crystals) has a positive effect on the precipitation formation process, as we established previously.

The results of research experiments on the artificial ionization of air influence on the droplets formation processes showed that at a field strength of $0.05 \mathrm{kV} \cdot \mathrm{m}^{-1}$, the diffused light intensity and vapour deposition time are out of sync with the background mode data (microwave radiation absence) within the statistical error of experiment. Analyzing the water vapour deposition time data (curve 2, Figure 10), it should be noted that an abrupt reducing the deposition time can be observed at values of $\mathrm{E} \geq 0.5 \mathrm{kV} \cdot \mathrm{m}^{-1}$, which allows concluding on non-rationality of using radiators providing for less field strength in the troposphere area for artificial precipitation forming.

The electromagnetic way of artificial precipitation formation is of a high potential yet it requires additional experimental research under the actual conditions.

\section{CONCLUSIONS}

The paper introduces a new method for emergency situations consequences with chemical and radioactive substances emission into the atmosphere elimination using artificial initiation of precipitation over an atmospheric pollution zone. To initiate precipitation under unfavorable meteorological conditions, chemical and electrophysical methods of influencing the processes of droplet formation have been proposed.

New pyrotechnic compositions have been developed with the addition of up to $60 \% \mathrm{KOH}$ or $\mathrm{NaOH}$, which are able to actively neutralize acidic pollution of the atmosphere and hazardous emissions of chlorine and phosgene.

The possibility of water vapour condensation at a partial air ionization by electromagnetic irradiation has been theoretically and experimentally established in the paper. It has been shown experimentally that artificial droplet formation in the atmosphere occurs at a field strength of $\mathrm{E} \geq$ $0.5 \mathrm{kV} \mathrm{m}^{-1}$. A method of focusing electromagnetic radiation from several ground-based sources has been proposed. Using this approach will reduce the required power of a single emitter.

\section{REFERENCES}

[1] Thibodeaux, L.J. Mackay, D. (2010) Chemical Mass Transport in the Environment. CRC Press, 631. https://doi.org/10.1201/b10262

[2] Atkinson, R. (2000) Atmospheric chemistry of VOCs and $\mathrm{NO}_{\mathrm{x}}$. Atmos. Environ, 34(12-24): 2063-2101.

[3] Brennan, W.J., Schultz, P.A. (2009) Atmospheric Aerosol Properties and Climate Impacts. U.S. Climate Change Science Program Synthesis and Assessment Product, 128.

[4] Artiola, J., Papper, I., Bruseau, M. (2004) Environmental Monitoring and Characterization. Elsevier Science \& Technology Books, 27.

[5] Philippin, S., Laj, P., Putaud, J.P., Wiedensohler, A., De Leeuw, G. (2009). EUSAAR An unprecedented network of aerosol observation in Europe. Earozoru Kenkyu. 24(2): 78-83. https://doi.org/10.11203/jar.24.78

[6] Kluska, K., Piotrowicz, K., Kasprzyk, I. (2020) The impact of rainfall on the diurnal patterns of atmospheric pollen concentrations. Agricultural and Forest Meteorology, 291: 108042. https://doi.org/10.1016/j.agrformet.2020.108042

[7] Yoschenko, V.I., Kashparov, V.A., Protsak, V.P., Lundin, S.M., Levchuk, S.E., Kadygrib, A.M., Tschiersch, J. (2006). Resuspension and redistribution of radionuclides during grassland and forest fires in the Chernobyl exclusion zone: Part I. Fire experiments. Journal of Environmental Radioactivity, 86(2): 143-163. https://doi.org/10.1016/j.jenvrad.2005.08.003

[8] Yamauchi, M., Takeda, M., Makino, M., Owada, T., Miyagi, I. (2012). Settlement process of radioactive dust to the ground inferred from the atmospheric electric field measurement. Ann. Geophys, 30(1): 49-56. https://doi.org/10.5194/angeo-30-49-2012 
[9] Steinhauser, G., Brandl, A., Johnson, T.E. (2014). Comparison of the Chernobyl and Fukushima nuclear accidents: A review of the environmental impacts. Science of the Total Environment, 470-471: 800-817. https://doi.org/10.1016/j.scitotenv.2013.10.029

[10] Guidelines for Mass Casualty Decontamination During a HAZMAT/ Weapon of Mass Destruction Incident. (2016). Volumes I and II. U.S. Army Chemical, Biological, Radiological and Nuclear School. USA. 136p. https://www.nfpa.org/ /media/files/news-and-research/ resources/external-links/firstresponders/decontamination/ ecbc guide masscasualtydecontam 0813.pdf?la=en

[11] Loosmore, G.A., Cederwall, R.T. (2004). Precipitation scavenging of atmospheric aerosols for emergency response applications: Testing an updated model with new real-time data. Atmospheric Environment, 38(7): 993-1003.

https://doi.org/10.1016/j.atmosenv.2003.10.055

[12] Xue, L., Hashimoto, A., Murakami, M., Rasmussen, R., Tessendorf, S.A., Breed, D., Blestrud, D. (2013). Implementation of a silver iodide cloud-seeding parameterization in WRF. Part I: Model description and idealized 2D sensitivity tests. Journal of Applied Meteorology and Climatology, 52(6): 1433-1457. https://doi.org/10.1175/JAMC-D-12-0148.1

[13] Silverman, B.A., Sukarnjanaset, W. (2000). Results of the Thailand warm-cloud hygroscopic particle seeding experiment. Journal of Applied Meteorology and Climatology, 39(7): 1160-1175. https://doi.org/10.1175/15200450(2000)039<1160:ROTTWC $>2.0 . \mathrm{CO} ; 2$

[14] Amiranashvili, A., Chikhladze, V., Dzodzuashvili, U.V., Ghlonti, N.Y., Sauri, I.P. (2015). Reconstruction of AntiHail System in Kakheti (Georgia). Journal of the Georgian Geophysical Society, Issue B. Physics of Atmosphere, Ocean and Space Plasma, 18: 92-106.

[15] Jin, R., Wang, W., Song, W., Wang, Z. (2012). Tianjin precipitation feature and analysis of weather modification influence. In Proceedings of the 11th WMO Scientific Conference on Weather Modification, Bali, Indonesia, pp. 127-132.

[16] Rosenfeld, D., Axisa, D., Woodley, W.L., Lahav, R. (2010). A quest for effective hygroscopic cloud seeding. Journal of Applied Meteorology and Climatology, 49(7): 1548-1562. https://doi.org/10.1175/2010JAMC2307.1

[17] Martinez-Castro, D., Pérez-Sanchez, C.A., Koloskov, B.P., Korneev, V.V., Petrov, V.V., Struinin, M., Novo-
Cuervo, S. (2011). Randomized convective cloud seeding experiment in extended areas in Cuba (EXPAREX). Revista Brasileira de Meteorologia, 26: 515-528.

[18] Guo, X., Fu, D., Li, X., Hu, Z., Lei, H., Xiao, H., Hong, Y. (2015). Advances in cloud physics and weather modification in China. Advances in Atmospheric Sciences, 32(2): 230-249. https://doi.org/10.1007/s00376-014-0006-9

[19] Sandhyavitri, A., Perdana, M.A., Sutikno, S., Widodo, F.H. (2018). The roles of weather modification technology in mitigation of the peat fires during a period of dry season in Bengkalis, Indonesia. In IOP Conference Series: Materials Science and Engineering, 309(1): 012016 https://doi.org/10.1088/1757$899 X / 309 / 1 / 012016$

[20] Rosenfeld, D. (2007). New insights to cloud seeding for enhancing precipitation and for hail suppression. The Journal of Weather Modification, 39(1): 61-69.

[21] Crowley, J.N., Ammann, M., Cox, R.A., Hynes, R.G., Jenkin, M.E., Mellouki, A., Wallington, T.J. (2010). Evaluated kinetic and photochemical data for atmospheric chemistry: Volume V-heterogeneous reactions on solid substrates. Atmospheric Chemistry and Physics, 10(18): 9059-9223. https://doi.org/10.5194/acp-10-9059-2010

[22] Attarakih, M., Abu-Khader, M., Bart, H.J. (2013). Dynamic analysis and control of sieve tray gas absorption column using MATALB and SIMULINK. Applied Soft Computing, 13(2): 1152-1169. https://doi.org/10.1016/j.asoc.2012.10.011

[23] Zikova, N., Zdimal, V. (2016). Precipitation scavenging of aerosol particles at a rural site in the Czech Republic. Tellus B: Chemical and Physical Meteorology, 68(1): 27343. https://doi.org/10.3402/tellusb.v68.27343

[24] Semko, A.N., Beskrovnaya, M.V., Vinogradov, S.A., Hritsina, I.N., Yagudina, N.I. (2014). The usage of high speed impulse liquid jets for putting out gas blowouts. Journal of Theoretical and Applied Mechanics, 52(3): 655-664.

[25] Gurevich, A.V., Borisov, N.D., Milikh, G.M., Book, D.L. (2018). Artificial Emission of Ionized Regions: Artificially Ionized Regions in the Atmosphere. https://doi.org/10.1201/9780203743287-6.

[26] Sinnhuber, M., Funke, B. (2020). Energetic electron precipitation into the atmosphere. In The Dynamic Loss of Earth's Radiation Belts, 279-321. https://doi.org/10.1016/B978-0-12-813371-2.00009-3 